\title{
Preventing Favism by Selecting Faba Bean Mutants Using Molecular Markers
}

AUTHOR: Melody Song

10.17975/sfj-2017-001

SCHOOL: Evan Hardy Collegiate Institute

Editor's note: This project was awarded second place within the Sanofi Biogenius Canada competition.

\section{Abstract}

Faba bean (Vicia faba) is an ancient legume species known for its high protein content. The usage and consumption of the faba bean is limited by a glycoside, vicine-convicine (VC). Consumption of VC causes haemolytic anemia in individuals with the genetic condition called favism. Faba beans with low VC concentration are opening the possibility of reduction of favism disease, but there are many challenges in analyzing VC concentration. The objective of this study was to develop expressed sequence tag (EST) markers that can differentiate between low VC content (LVC) and high VC content (HVC) faba bean genotypes. Three single nucleotide polymorphisms (SNPs) were discovered that distinguished between LVC and HVC genotypes. The SNPs were validated using Kompetitive Allele Specific PCR (KASP) and mass spectrometry phenotyping. Molecular marker SNP 316 (Intron of Medtr2g009270 at 1,851,012 bp) was the most successful marker in differentiating between LVC, HVC, and heterozygous faba bean genotypes. This marker has applications in seed selection and acceleration of breeding programs, which is the first step towards allowing all consumers concerned with the effects of favism to enjoy the nutritional value of faba bean.

\section{Key Words}

Faba bean; Vicine-convicine (VC); Expressed Sequence Tag (EST); Molecular Marker; Single Nucleotide Polymorphism (SNP)

\section{Introduction}

The faba bean is an ancient legume species known for its high protein content, nitrogen-fixation ability and adaptive capability in cool climates (1). It provides many nutritional benefits with high quantities of carbohydrates, minerals, vitamins, and dietary fiber. The faba bean is also a rich source of levo-3,4-dihydroxy-phenylalanine (L-DOPA), a major ingredient in medication for Parkinson's disease management (2). However, the use and consumption of the faba bean is limited by the pyrimidine glycosides: vicine and convicine. The compound, vicine-convicine (VC), causes acute haemolytic anemia in individuals with glucose-6-phosphate dehydrogenase deficiency by triggering oxidative stress, leading to premature destruction of red blood cells. This disease, also known as favism, affects more than 400 million people worldwide (3). Mutant faba beans with low VC concentrations have been discovered, however there are many challenges in quantifying VC levels. This includes a lack of candidate genes for $\mathrm{VC}$, unknown biosynthetic pathways and a massive genome of nearly $13 \mathrm{Gbp}$.

Expressed sequence tag (EST) sequences are short genetic sequences, which can be used for gene sequence determination, through the creation of markers, to select traits of interest. ESTbased molecular markers are an efficient, quick and relatively inexpensive genetic method of selecting promising faba bean lines. Six EST sequences associated with VC synthesis have been published (4). The VC QTL gene coding region has been mapped with flanking SNP markers, developed using the model species Medicago truncatula (5). The main objective of this study was to develop successful EST-based molecular markers to differentiate between LVC and HVC faba bean genotypes.

\section{Materials}

Table 1: EST candidate sequences (4).

\begin{tabular}{|l|l|l|l|l|l|}
\hline EST Sequences \\
\hline Contig 1899 & Contig 299 & Contig 412 & Contig 4518 & Contig 4684 & Contig 4824 \\
\hline
\end{tabular}

Table 2: Four Faba bean genotypes used for sequencing.

\begin{tabular}{|l|l|l|}
\hline Name & VC Content & Origin \\
\hline CDC Snowdrop & HVC & Canada \\
\hline L_43 & HVC & Bangladesh \\
\hline ILB938/2 & HVC & Ecuador \\
\hline Melodie/2 & LVC & France \\
\hline
\end{tabular}

\section{Methodology}

\section{Sequencing and Marker Design}

Six candidate EST sequences associated with VC were identified (see supplemental Table 1). EST sequences were aligned with 
Table 3: Variety of 52 faba bean genotypes used for marker validation

\begin{tabular}{|l|l|l|l|}
\hline \multicolumn{2}{|l|}{52 faba bean genotypes } & FBR-06-137 & Disco/2* A01155 \\
\hline FBR-06-1 & FBR-06-90 & FBR-06-138 & Disco/2 * CDC Snowdrop \\
\hline FBR-06-2 & FBR-06-91 & FBR-06-185 & Divine \\
\hline FBR-06-6 & FBR-06-93 & FBR-06-199 & Fabelle \\
\hline FBR-06-9 & FBR-06-94 & 1778 & Gloria \\
\hline FBR-06-10 & FBR-06-95 & $346-10$ & ILB938/2 \\
\hline FBR-06-11 & FBR-06-107 & A01155 & L-43 \\
\hline FBR-06-12 & FBR-06-114 & A01155* Disco/2 & L-170 \\
\hline FBR-06-18 & FBR-06-115 & AF11212 & L-175 \\
\hline FBR-06-21 & FBR-06-116 & AF11212 * Disco/2 & Medina \\
\hline FBR-06-40 & FBR-06-121 & CDC Snowdrop & Melodie/2 \\
\hline FBR-06-48 & FBR-06-128 & CDC Snowdrop * A01155 & P47-1 \\
\hline FBR-06-79 & FBR-06-129 & Disco/2 & SSNS-1 \\
\hline FBR-06-87 & FBR-06-136 & & \\
\hline
\end{tabular}

NCBI Blast. ESTs were also aligned to the model species Medicago truncatula using Phytozome software. Contig 4518 was matched to Medtr2g009270 gene which was found in chromosome 2 of the Medicago truncatula genome (Figure 1), and identified within the VC QTL gene coding region. Using Contig 4518, sequencing primers were designed for marker development (Table 4).

\section{Marker Development}

To identify SNPs, four faba bean genotypes were sequenced (Table 2). DNA was gathered from seed cotyledons using Micro-CTAB DNA Extraction and was quantified with Nanodrop. Polymerase chain reaction (PCR) amplified the DNA and EST-based sequencing primers (Table 4 ), with 40 cycles at $94^{\circ} \mathrm{C}$ for $60 \mathrm{~s}, 56^{\circ} \mathrm{C}$ for $60 \mathrm{~s}$ and $72^{\circ} \mathrm{C}$ for $60 \mathrm{~s}$. PCR products were visualized on a $2 \%$ agarose gel and DNA fragments were extracted using QIAquick Gel Extrac- tion Kit. The purified DNA was sequenced using Sanger sequencing with Eurofins Genomics. The sequences of the four faba bean genotypes were aligned using BioEdit Sequence Alignment Editor software and three SNPs were identified, distinguishing between LVC and HVC genotypes. The placement of the SNPs were located on the Medtr2g009270 gene by comparing the SNP sequences to the original EST sequence using Phytozome software. The exact basepair location on the Medicago truncatula genome was identified by analyzing the exons and introns on Medtr2g009270. Primers were designed for the molecular marker validation based on the flanking DNA sequences of the SNPs (Table 5).

\section{Marker Validation}

KASP assay was used for the SNP genotyping molecular marker validation. The markers were validated with 52 known HVC and

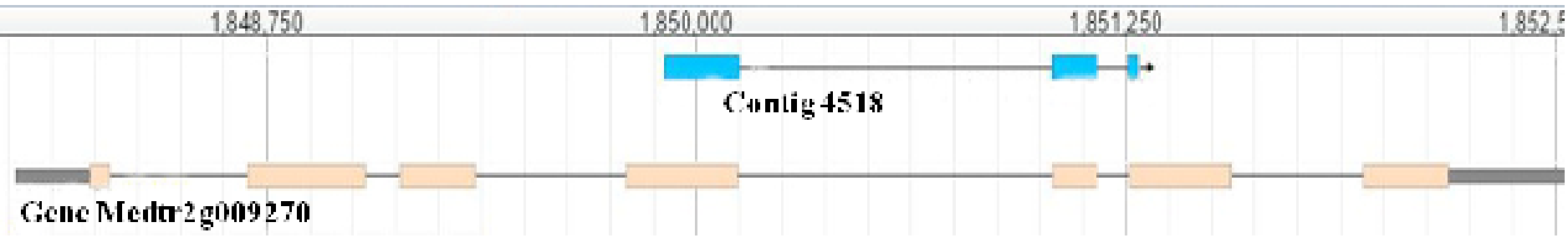

Figure 1: Comparative Sequence Alignment with Contig 4518 and Medtr2g009270 (Phytozome) 
Table 4: Sequencing primers for molecular marker development.

\begin{tabular}{|l|l|}
\hline Contig & Sequencing Primers \\
\hline & $\begin{array}{l}\text { Forward Primer: } \\
\text { ATTTTCCCAGTAAAATACAGTGAAG }\end{array}$ \\
\hline & Reverse Primer: ACATCTTCTCCATCTCCAAGG \\
\hline
\end{tabular}

LVC faba bean genotypes (Table 3). DNA was extracted with $\mathrm{NaOH}$ Treatment of Plant Seeds for quick DNA release for the KASP assay. Molecular marker KASP primers and DNA were prepared with KBioscience KASP Genotyping Quick Start Guide. KASP assays were run with $10.14 \mu \mathrm{l}$ of reaction mix, including $5 \mu \mathrm{l}$ of DNA and $5.14 \mu \mathrm{l}$ of Master-Mix (KASP Genotyping Quick Start Guide). The PCR conditions for KASP marker assay was $95^{\circ} \mathrm{C}$ for $15 \mathrm{~min}$, followed by 10 cycles of $94^{\circ} \mathrm{C}$ for $20 \mathrm{~s}$ and $65^{\circ} \mathrm{C}$ for 2 min, then followed by 40 cycles of $94^{\circ} \mathrm{C}$ for $20 \mathrm{~s}$ and $57^{\circ} \mathrm{C}$ for $1 \mathrm{~min}$. PCR readings were performed with AB Applied Biosystems Real-Time PCR System. Online software, Step-One, was used in the data analysis of the samples. The same 52 faba bean seed samples which were used in the KASP SNP genotyping were ground into powder for phenotyping. The phenotypic data was gathered using mass spectrometry screening.

\section{Results}

\section{SNP Discovery}

Three SNPs were identified with Sanger sequencing. SNP $316[\mathrm{G} / \mathrm{C}]$ was identified in an intron at $1,851,012 \mathrm{bp}$ on the Medtr2g009270 gene, SNP 384 [T/A] was identified in an exon at $1,851,080 \mathrm{bp}$ and SNP $423[\mathrm{C} / \mathrm{T}]$ was identified in an exon at

\section{$1,851,119$ bp. (Figure 2)}

\section{SNP Marker Validation}

The SNPs were validated using KASP assays and mass spectrometry. KASP assays were developed to test the three SNPs using 52 faba bean genotypes varying in VC concentrations. For SNP 316 , two trials of KASP assay were run with a $98 \%$ similarity, the assays displayed accurate negative controls and distinct clusters. Blue clusters were associated with HVC and red clusters were associated with LVC. KASP Assay could also distinguish heterozygous samples: Gloria, P47-1-A, CDC Snowdrop x A01155, AF11212 x Disco/2 and Disco/2 $x$ CDC Snowdrop. There were two outliers during the two trials, FBR-06-114 and AF11212 (Figure 3). KASP assays for SNP 384 and 423 displayed a greater scatter of the samples and negative control.

Mass spectrometry phenotyping was performed to confirm the genotyping results. The same 52 seed samples were used and the results were compared between both genotyping and phenotyping validation methods. Phenotyping could not distinguish the five heterozygous samples from HVC and LVC. 44 out of 47 samples shared the exact same results, and there were three genotypes that did not

Table 5: KASP molecular marker validation primers.

\begin{tabular}{|l|l|l|l|}
\hline Locus & Marker & Gene & Sequencing primer \\
\hline Chr 2 & SNP 316 & Medtr2g009270 & $\begin{array}{l}\text { Forward1: } \\
\text { GAAGGTGACCAAGTTCATGCTTTGTTTTTAATGTAATTTACTGTCTATGAT } \\
\text { AG }\end{array}$ \\
\hline & & & $\begin{array}{l}\text { Forward2: } \\
\text { GAAGGTCGGAGTCAACGGATTTGTTTTTAATGTAATTTACTGTCTATGAT } \\
\text { AC }\end{array}$ \\
\hline & & & Common: CTATATCTGACATAGCAACAAATAAGTGTT \\
\hline Chr 2 & SNP 384 & Medtr2g009270 & $\begin{array}{l}\text { Forward1: } \\
\text { GAAGGTGACCAAGTTCATGCTGACATATTAATAGAACGCTCTTCTGCT }\end{array}$ \\
\hline & & & $\begin{array}{l}\text { Forward2: } \\
\text { GAAGGTCGGAGTCAACGGATTGACATATTAATAGAACGCTCTTCTGCA }\end{array}$ \\
\hline Chr 2 & SNP 423 & Medtr2g009270 & $\begin{array}{l}\text { Forward1: } \\
\text { GAAGGTGACCAAGTTCATGCTCAGTGGGGGAAATTCACATCATAC }\end{array}$ \\
\hline & & & $\begin{array}{l}\text { Forward2: } \\
\text { GAAGGTCGGAGTCAACGGATTCTCAGTGGGGGAAATTCACATCATAT }\end{array}$ \\
\hline & & & \begin{tabular}{l} 
Common: CCATTGCAATATGCTCAATCCCGTCTA \\
\hline
\end{tabular} \\
\hline
\end{tabular}




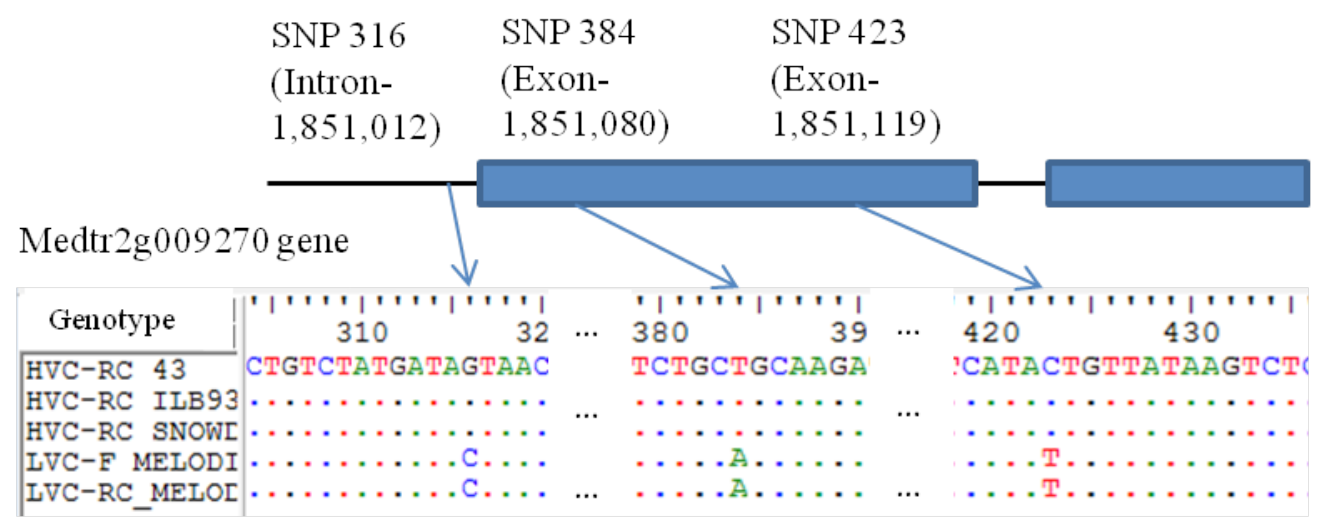

Figure 2: Placement of SNPS on Medtr2g009270

match their phenotypes: SSNS-1, L 170 and L 175.

\section{Discussion}

The most successful molecular marker SNP 316 was found in an intron at 1,851,012 bp of the gene Medtr2g009270. Although, the SNP was identified within an intron, its success shows that the SNP is closely linked to the gene of interest. Through KASP marker validation, two trials of SNP 316 were run to determine accuracy of the molecular marker. There was a 94\% accuracy between genotyping and phenotyping results; three genotypes differed from their phenotypic data: SSNS-1, L_170, and L_175. A number of factors may have led to these differences including phenotyping/technical errors or inaccurate seed source. The molecular marker was able to identify heterozygous samples, therefore the molecular marker SNP 316 demonstrates an advantage over phenotyping as it can be used to distinguish heterozygous genotypes. In the KASP marker validation, there were also two outliers, FBR-06-114 and AF11212, which were potentially caused by not having adequate amounts of DNA in the KASP reaction.

For SNP 384, the KASP assay had inaccurate negative controls and poor formulation of distinct clusters. For SNP 423, the KASP assay displayed a large and imprecise cluster of heterozygous samples. The unsuccessful marker validations may have been caused by nonspecific primer binding.
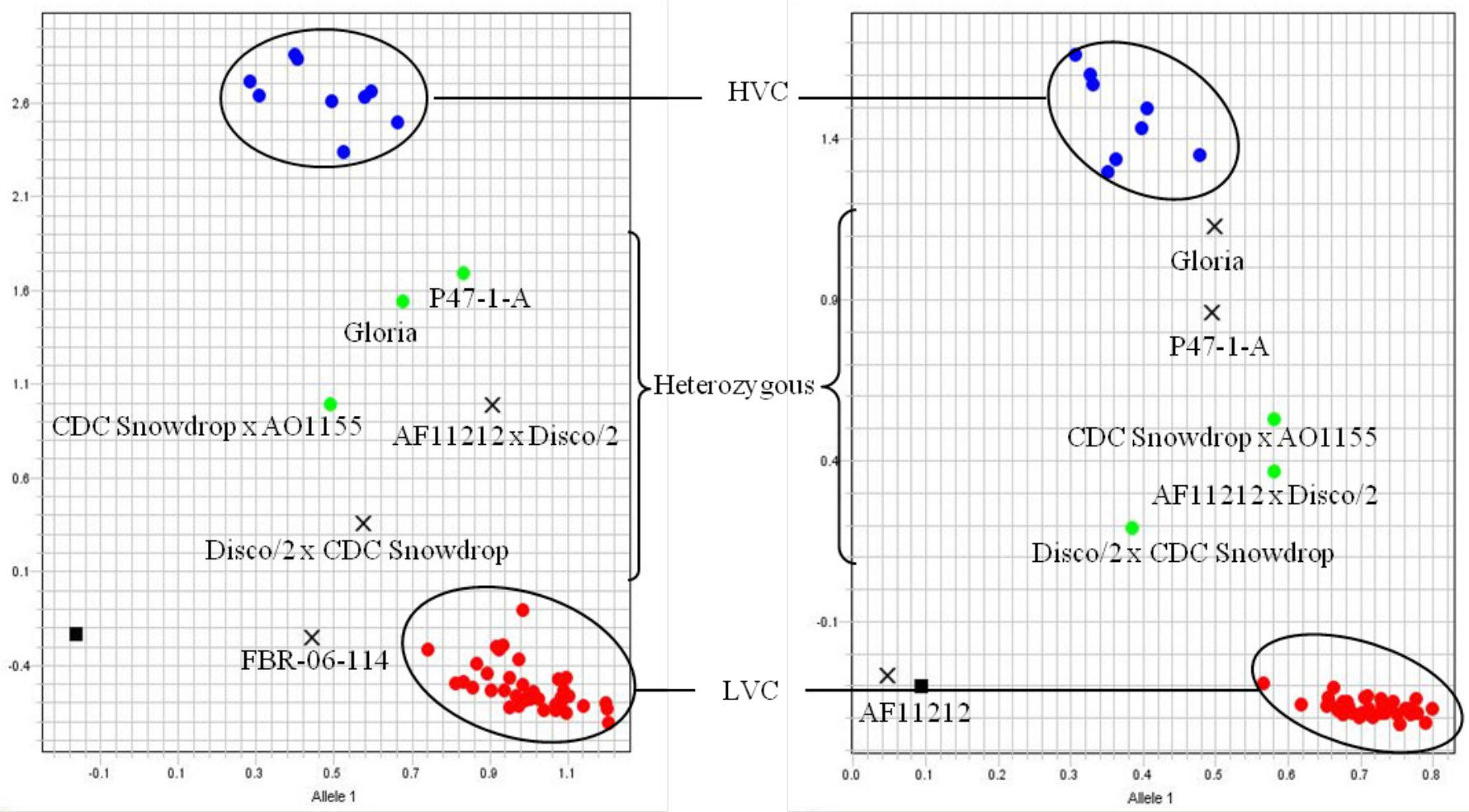

Figure 3: KASP Assay Results for SNP 316. 
Future directions include the development of a diagnostic seed test through the optimization of the molecular markers for largescale use in detecting VC content leading to acceleration of breeding programs to create low VC faba beans for human and livestock consumption.

\section{Conclusions}

Contig 4518 and its homologous gene Medtr2g009270 was successful for the development of the EST-based molecular marker. Molecular Marker SNP 316 at 1,851,012 bp in an intron of Medtr2g009270 was identified as the most successful EST-based molecular marker with accurate ability to differentiate between known HVC and LVC genotypes and distinguish heterozygous genotypes through KASP assay. The molecular marker is superior to other methods as it is inexpensive, quick and requires fewer re- sources. Ultimately, the molecular marker can lead to increased consumer demand and health through efficient seed selection of LVC faba beans.

\section{Acknowledgements}

I would like to thank the Department of Plant Sciences, University of Saskatchewan, Dr. Kirstin Bett and Dr. Hamid Khazaei for their mentorship, Dr. Albert Vandenberg for supporting this project, Mr. Rob Stonehouse for guidance in primer design and sequencing, and Dr. Randy Purves for phenotyping results. Thank you to the Sanofi Biogenius Canada for encouraging students to be involved in the field of biotechnology.

This research was supported by the funding from Saskatchewan Pulse Growers.

\section{References}

1. Duc G. Faba bean (Vicia faba L.). Field Crops Res. 1997 July;53(1-3):99-109.

2. Goyoaga C, Burbano C, Cuadrado C, Varela A, Guillamón E, Pedrosa M, Muzquiz M. (2008). Content and distribution of vicine, convicine and 1-DOPA during germination and seedling growth of two Vicia faba L. varieties. Eur Food Res Technol. 2008 April;227(5):1537-1542.

3. Cappellini M.D, Fiorelli G. Glucose-6-phosphate dehydrogenase deficiency. Lancet. 2008 Jan 5;371(9606):64-74. doi:10.1016/S01406736(08)60073-2.

4. Ray H, Bock C, Georges F. Faba bean: transcriptome analysis from etiolated seedling and developing seed coat of key cultivars for synthesis of proanthocyanidins, phytate, raffinose family oligosaccharides, vicine, and convicine. Plant Genome. 2015 March;8(1). doi:10.3835/plantgenome2014.07.0028

5. Khazaei H, O’Sullivan D, Jones H, Pitts N, Sillanpää M, Pärssinen P, Stoddard F. Flanking SNP markers for vicine-convicine concentration in faba bean (Vicia faba L.). Mol Breed. 2015 Jan;35(1):38-44. doi:10.1007/s11032-015-0214-8

\section{REVIEW}

\section{by Dr. Jim Todd,}

\section{Ontario Ministry of Agriculture, Food and Rural Affairs}

The research presented in this paper was fundamentally sound. Studies such as this one provide the necessary tools to aid future efforts to breed low content VC faba beans, the development of which would be beneficial to many sectors of the agricultural industry. I was impressed by the quality of the work done by the author. In general, the paper was well written, but would benefit from additional detail on the method used and the results of the phenotyping study. Future studies to identify additional SNP markers would be useful. It would also be interesting to determine if the low VC containing faba bean lines have different agronomic performance compared to the high VC containing lines. 